\title{
Pixel-level tunable liquid crystal lenses for auto-stereoscopic display
}

\author{
Kun $\mathrm{Li}^{\mathrm{a}}$, Brian Robertson ${ }^{\mathrm{a}}$, Mike Pivnenko ${ }^{\mathrm{a}}$, Daping Chu ${ }^{\mathrm{a} *}$, Jiong Zhou ${ }^{\mathrm{b}}, \mathrm{Jun}_{\mathrm{Yao}}{ }^{\mathrm{b}}$ \\ ${ }^{a}$ Photonics \& Sensors Group, Dept. of Engineering, Univ. of Cambridge, 9 JJ Thomson Avenue, \\ Cambridge, CB3 0FA, United Kingdom; ${ }^{b}$ Huawei Technologies Co., Ltd. Bantian, Longgang \\ District, Shenzhen, 518129 China
}

\begin{abstract}
Mobile video and gaming are now widely used, and delivery of a glass-free 3D experience is of both research and development interest. The key drawbacks of a conventional 3D display based on a static lenticular lenslet array and parallax barriers are low resolution, limited viewing angle and reduced brightness, mainly because of the need of multiple-pixels for each object point.

This study describes the concept and performance of pixel-level cylindrical liquid crystal (LC) lenses, which are designed to steer light to the left and right eye sequentially to form stereo parallax. The width of the LC lenses can be as small as $20-30 \mu \mathrm{m}$, so that the associated auto-stereoscopic display will have the same resolution as the 2D display panel in use. Such a thin sheet of tunable LC lens array can be applied directly on existing mobile displays, and can deliver 3D viewing experience while maintaining $2 \mathrm{D}$ viewing capability.

Transparent electrodes were laser patterned to achieve the single pixel lens resolution, and a high birefringent LC material was used to realise a large diffraction angle for a wide field of view. Simulation was carried out to model the intensity profile at the viewing plane and optimise the lens array based on the measured LC phase profile. The measured viewing angle and intensity profile were compared with the simulation results.
\end{abstract}

Keywords: auto-stereoscopic display, liquid crystal lens, pixel-level, tunable

\section{INTRODUCTION}

A mobile phone with a glass-free 3D experience would be a very attractive feature if the 3D image has the same resolution as the original 2D display and if the feature can be switched back to $2 \mathrm{D}$ when needed to view text [1][2]. The conventional auto-stereoscopic display based on spatial multiplexing with lenticular arrays and parallax barriers are not suitable because they sacrifice the image resolution, brightness, and they are not switchable. Other techniques like holography takes too much computing power and is currently only practical for still images [3]. A newly developed guided-wave illumination device based on light-emitting diodes permits the rendering of high-resolution, full-parallax 3D images in a very wide view zone [4], but this technology has not been proved in a commercial application.

In this study, we describe a pixel-level tunable liquid crystal (LC) lens. The lens uses an electrically controlled LC layer to display truncated off-axis lenses or linear phase gradients that can reconfigurably deflect light from each display pixel through two or more angles in the time domain or simultaneously, thereby implementing an auto-stereoscopic display. Each LC lens pitch is of the same order as a typical mobile display pixel in the market, so that the full resolution of the $2 \mathrm{D}$ display is preserved after switching to the $3 \mathrm{D}$ mode. Such a lens sheet can also be integrated directly onto an existing mobile display.

LC optical devices have been previously used in adaptive optical systems to compensate for aberrations [5], and as spatial light modulators, active lenses, and bandpass filters [6]. An auto-stereoscopic 3D display using LC lenses was also proposed previously, where a multi-electrode driven scheme was utilized to form a refractive index curve closer to the ideal lens in order to reduce crosstalk [7]. However, the size of LC lens used in past systems were in range from a few hundred micrometers to a few millimeters, which does not match the $\sim 100 \mu \mathrm{m}$ pixel size of modern mobile phones.

*Correspondence: dpc31@cam.ac.uk

Advances in Display Technologies IV, edited by Liang-Chy Chien, Sin-Doo Lee,

Ming Hsien Wu, Proc. of SPIE Vol. 9005, 900505 - (c) 2014 SPIE

CCC code: $0277-786 X / 14 / \$ 18 \cdot$ doi: $10.1117 / 12.2039065$

Proc. of SPIE Vol. 9005 900505-1 
Two high birefringent nematic LCs were used in the study, LC1 and LC2. The Indium Tin Oxide (ITO) coated substrates used to form the LC cells were patterned with a laser. The phase profile of the LC lens was evaluated using transmissive optical microscopy, and the phase lens was simulated in ZEMAX to determine steering ability.

\section{LIQUID CRYSTAL LENS ARRAY}

The LC cylindrical lens was designed with a radius of $\sim 20 \mu \mathrm{m}$, and an array of LC lenses were fabricated by laser patterning of ITO coated soda lime substrates. A microscope image of the patterned substrate is shown in Figure 1. The ITO electrode is $\sim 10-12 \mu \mathrm{m}$ wide, and the distance between two neighbouring electrodes is $\sim 38-40 \mu \mathrm{m}$. We used a single mode nanosecond pulsed fibre laser (G3 SM-S00044_1, SPI Lasers, Southampton, UK.) working at 1062nm wavelength which achieved a $\sim 10 \mu \mathrm{m}$ resolution. The LC lens consisted of a LC layer sandwiched between the two display substrates and was made in a cleanroom environment. The glasses are $0.55 \mathrm{~mm}$ thick with a $\sim 20 \mathrm{~nm}$ thick ITO coating which provides $\sim 100 \Omega / \square$ sheet resistance.

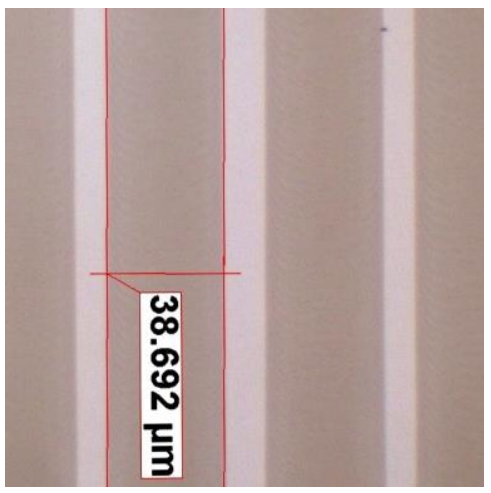

Figure 1. A microscope image of laser patterned ITO electrodes; the brighter areas are the ITO tracks.

The intensity profiles of the resulting LC lens array between cross polarizers are shown in Figure 2 at three driving voltages. The phase profiles were calculated for LC1 and LC2, and plotted in Figure 3 at varying driving voltages. The phase profile width or the LC lens pitch, defined as the distance between the $10 \%$ and $90 \%$ of the maximum phase value at that driving condition, increased with the higher driving voltages. The maximum lens pitch is about $20 \mu \mathrm{m}$ for both LCs, and the maximum phase difference is $\sim 8.3 \pi$ for LC1, and $\sim 14 \pi$ for LC2. The higher phase difference will provide a larger steering angle for a fixed lens radius. The measured phase curve was then used to simulate the steering ability of the LC lens.

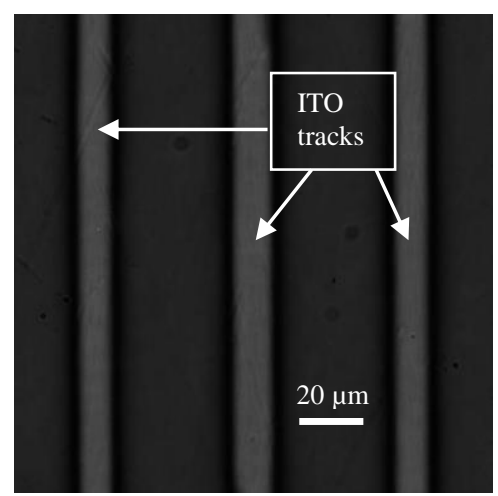

(a)

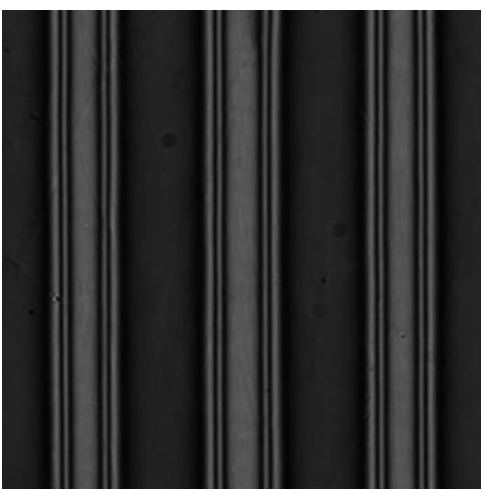

(b)

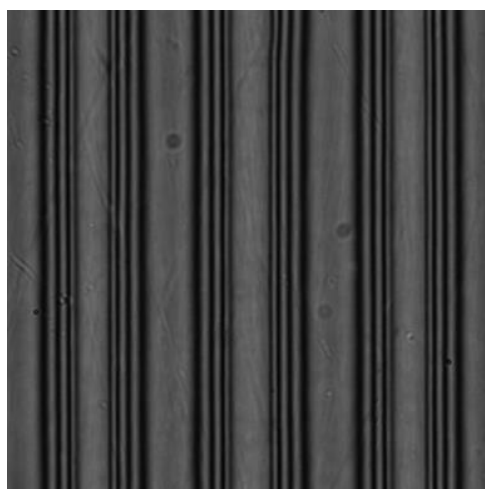

(c)

Figure 2. Grey scale intensity images of the LC lens array at a driving voltage of (a) $5 \mathrm{~V}$, (b) $7 \mathrm{~V}$ and (c) $12 \mathrm{~V}$ peak to peak for LC1. 


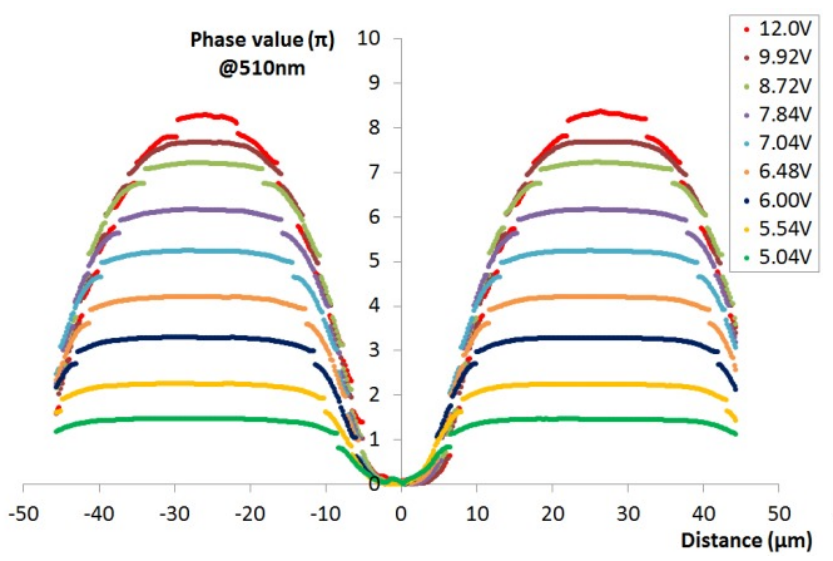

(a)

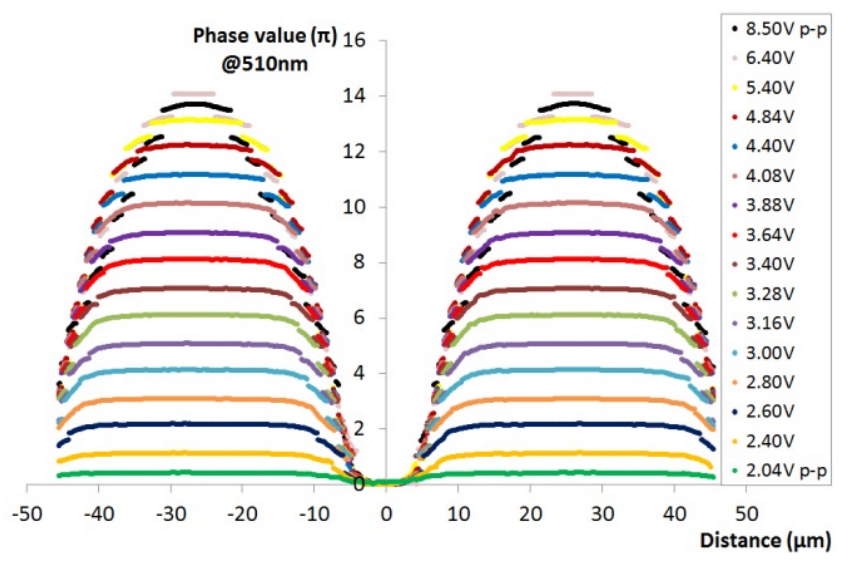

(b)

Figure 3. The calculated phase profiles at varying driving voltages applied to ITO electrodes for (a) LC1 and (b) LC2.

\section{SIMULATION}

The optics design software ZEMAX was used to compare a paraxial steering lens with a LC steering lens based on the measured LC phase profile. Physical optics propagation (POP) was used to calculate the light irradiance at the viewing plane $300 \mathrm{~mm}$ from the LC lens, the typical viewing distance of a mobile user. The input was a Top-hat intensity profile with a $10 \mu \mathrm{m}$ waist $(20 \mu \mathrm{m}$ diameter) at $510 \mathrm{~nm}$ wavelength to represent a sub-pixel.

The measured phase profile of the LC lens filled with LC2 material ( $14 \pi$ phase difference) was compared to two quadratic phase profiles (ideal paraxial lens) that give $11^{\circ}$ and $13^{\circ}$ steering angles respectively, as shown in Figure 4. The phase value of the paraxial lens was calculated based on the geometrical representation of a spherical lens, as shown

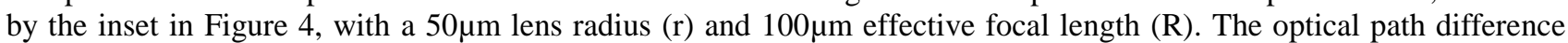
when light enters the lens at different distance from the chief ray was multiplied by $2 \pi / \lambda$ to give the phase value. A portion of the phase profile was then sectioned off the entire paraxial lens curve to give the required beam steering, with the steering angle selection based on a best fit practice.

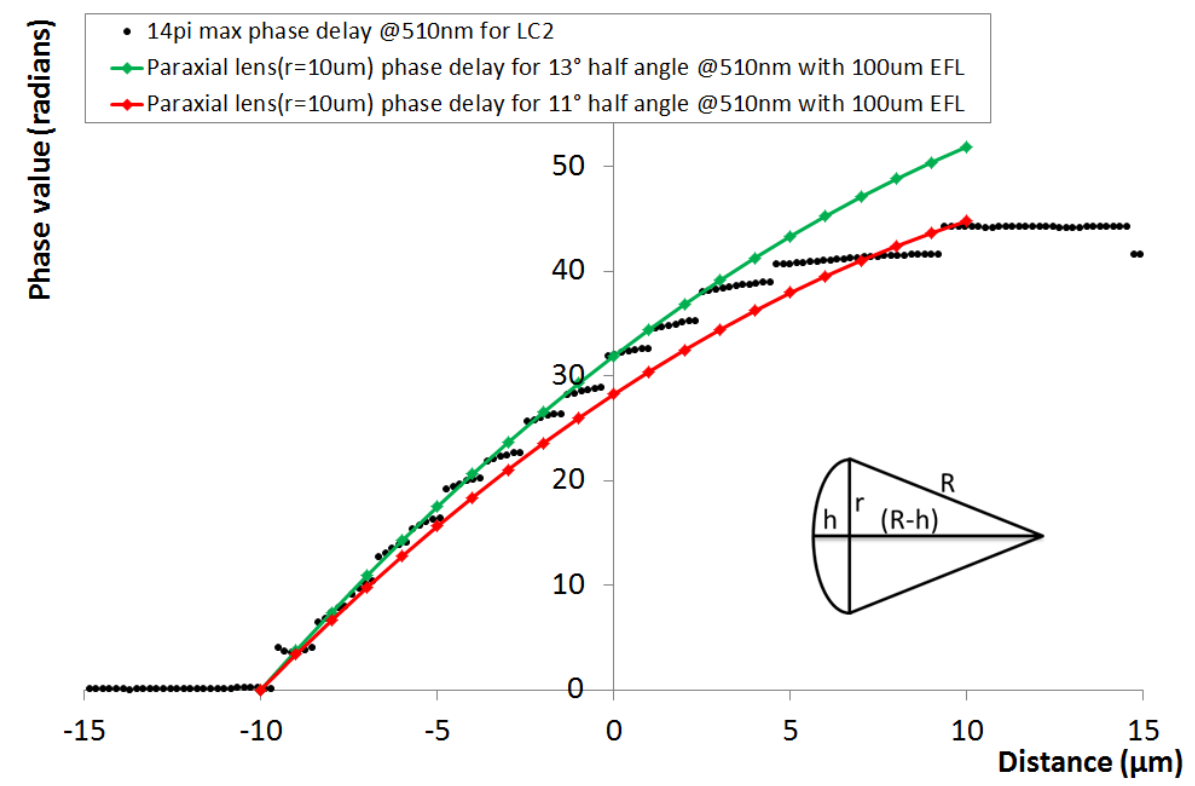

Figure 4. The phase curve comparison of two perfect steering lenses with $11^{\circ}$ and $13^{\circ}$ steering angles with the LC steering lens. 
As can be seen, the paraxial phase curve corresponding to a $13^{\circ}$ steering angle matches the LC lens phase profile very well from $-10 \mu \mathrm{m}$ up to a point at $+5 \mu \mathrm{m}$ distance, Figure 4 . Between $+5 \mu \mathrm{m}$ and $+10 \mu \mathrm{m}$, the LC lens phase curve fits between the two ideal phase curves corresponding to $11^{\circ}$ and $13^{\circ}$ steering angles. In this work we chose $13^{\circ}$ steering angle paraxial lens as the reference point for comparison due to the closer fit.

\subsection{Paraxial lens}

A paraxial cylindrical lens $(\mathrm{r}=50 \mu \mathrm{m})$ was defined in ZEMAX with a $100 \mu \mathrm{m}$ effective focal length in the y direction, and no lens power defined in the $\mathrm{x}$ direction. The source beam was decentred by $23 \mu \mathrm{m}$ in the $\mathrm{y}$ direction to give a $13^{\circ}$ steering angle. The output irradiance profile at the viewing plane (300 $\mathrm{mm}$ away from source) along with cross section irradiance profiles in the y directions are shown in Figure 5. The centre of the output beam is approximately 69 mm away from the optical axis confirming the $13^{\circ}$ steering angle. The $\mathrm{d}_{\mathrm{y}}$ value $(\sim 57 \mathrm{~mm})$, defined as the width of the beam when the irradiance value is above $1 / \mathrm{e}^{2}$ of the maximum value, was a result of both steering and diffraction. The output peak irradiance is $9.84 \times 10^{-7} \mathrm{~W} / \mathrm{mm}^{2}$ with the input value assumed to be $1 \mathrm{~W} / \mathrm{mm}^{2}$.

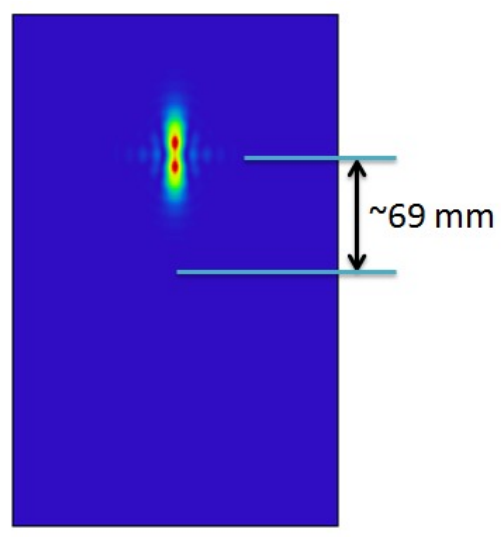

(a)

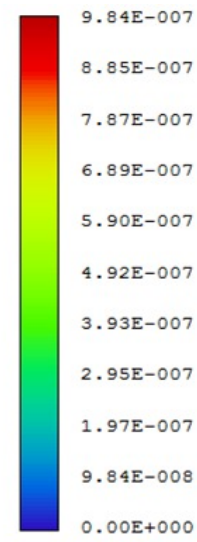

$.00 \mathrm{E}+000$

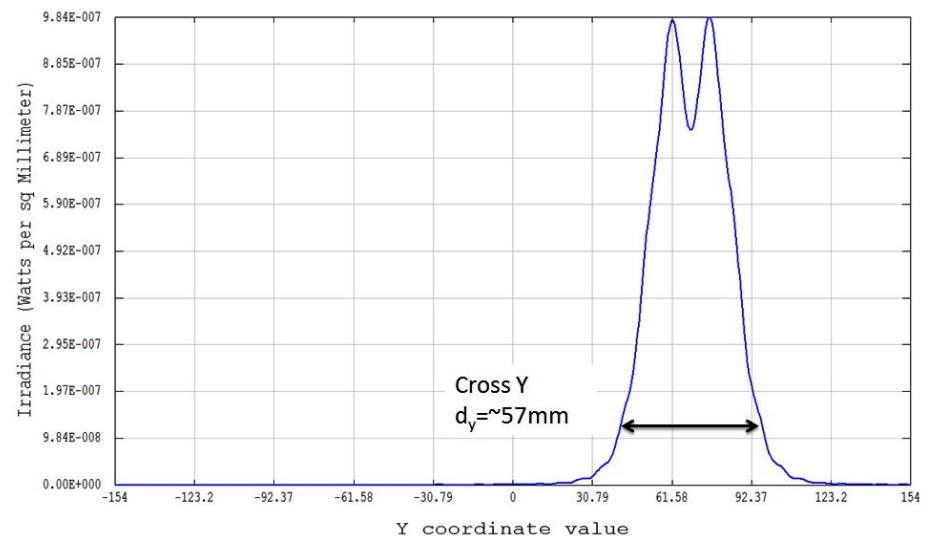

(b)

Figure 5. (a) The output beam irradiance profile at the viewing plane for the paraxial cylindrical lens. (b) The cross section profile in the $y$ directions, the $d_{y}$ is the diameter of the beam when the irradiance value is above $1 / \mathrm{e}^{2}$.

\subsection{LC lens}

In ZEMAX, an extended polynomial equation (Binary Optics 1 [8]) was used to represent the measured phase profile of the fabricated LC lens. This defines the continuous variation in the measured phase profile, $\delta$, as:

$$
\delta=M \sum_{i=1}^{N} A_{i} E_{i}(x, y)
$$

where $N$ is the number of polynomial coefficients in the series, $A_{i}$ the coefficient on the $i^{\text {th }}$ extended polynomial term, and $M$ the diffraction order. The phase profile of the LC lens can be represented as a series of polynomials in Origin $\left(\mathrm{OriginLab}^{\circledR}\right)$ data analysis software, Figure 6(a). The mathematical representation was nearly a perfect match to the original shape apart from the valleys of the phase profile. The coefficient of each polynomial term was then exported into ZEMAX to model the measured cylindrical LC lens. The green arrow indicates how a light beam would behave after passing through the steering part of the phase curve. The resulting phase profile of the LC lens as defined by Binary Optics 1 is shown in Figure 6(b) as fringe patterns in ZEMAX. 


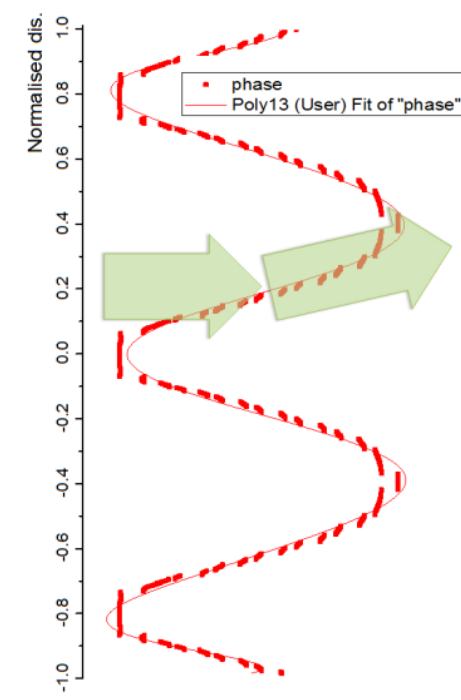

(a)
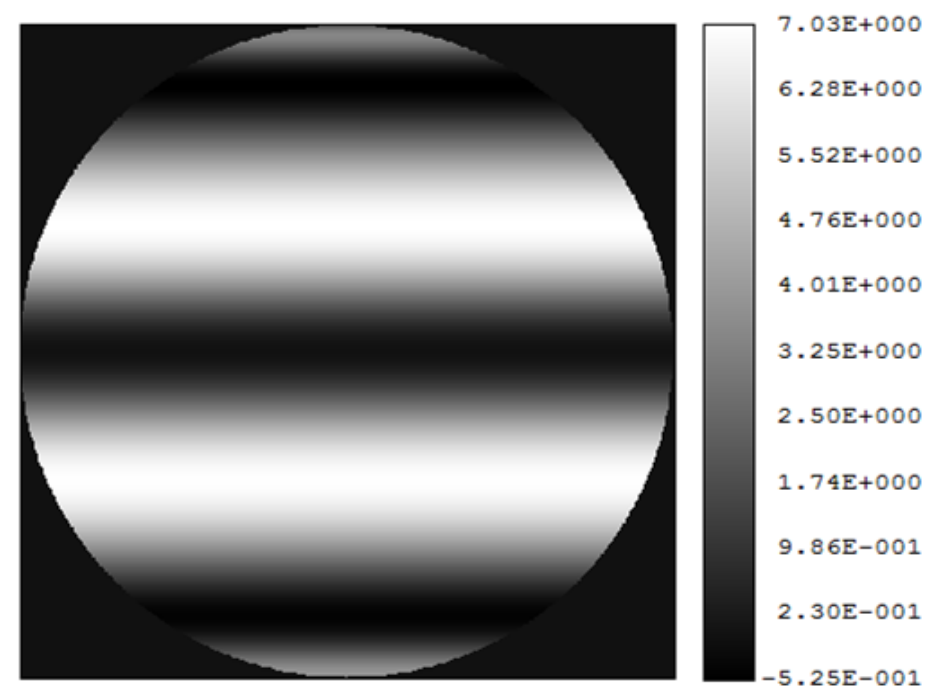

(b)

Figure 6. (a) The fitted curve of the phase profile (LC2 lens) with a 13-term polynomial, the green arrow shows the steering direction of the phase lens. (b) Phase distribution of the ZEMAX model used to simulate the LC lens, the units on the right are periods of $2 \pi$ radians each.

The same top-hat beam $(\mathrm{d}=20 \mu \mathrm{m})$ was applied to the extended polynomial definition of the measured LC lens, with a $126 \mu \mathrm{m}$ lens diameter to match the patterned electrode dimensions. The modeled phase lens behaves as a cylindrical LC lens to the input beam. The input beam was decentred by $13 \mu \mathrm{m}$ so that it aligns to the steering part of the lens, shown as the green arrow in Figure 6(a), and a POP analysis was performed. The output irradiance profile at the viewing plane (300 mm away from source) with the cross section irradiance profile in the y directions are shown, Figure 7 . The peak irradiance of the output is $\sim 68 \mathrm{~mm}$ away from the optical axis giving a $\sim 13^{\circ}$ steering angle. There is a dotted tail next to the central irradiance peak, thought to be caused by the input beam overfilling the LC lens. The subsequent beam clipping causes the observed diffraction pattern. The $\mathrm{d}_{\mathrm{y}}$ value $(\sim 60 \mathrm{~mm})$ was a result of LC lens steering and diffraction. The output peak irradiance is $1.56 \times 10^{-6} \mathrm{~W} / \mathrm{mm}^{2}$ with the input value assumed to be $1 \mathrm{~W} / \mathrm{mm}^{2}$.

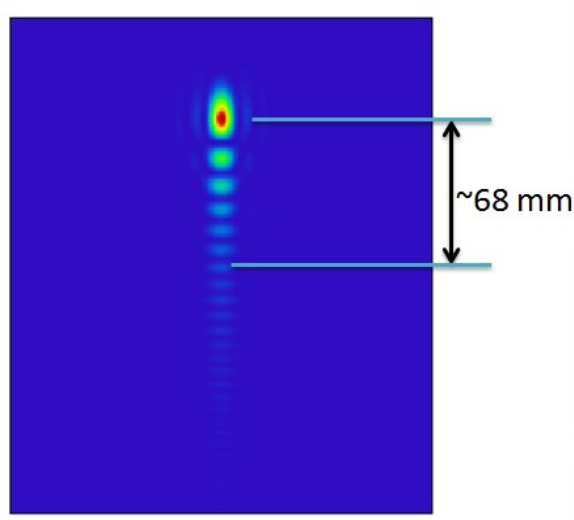

(a)

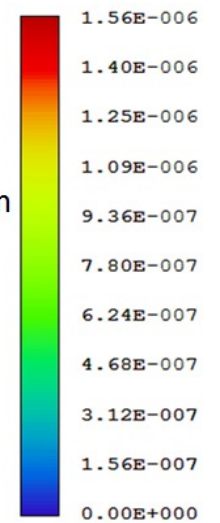

$0.00 \mathrm{E}+000$

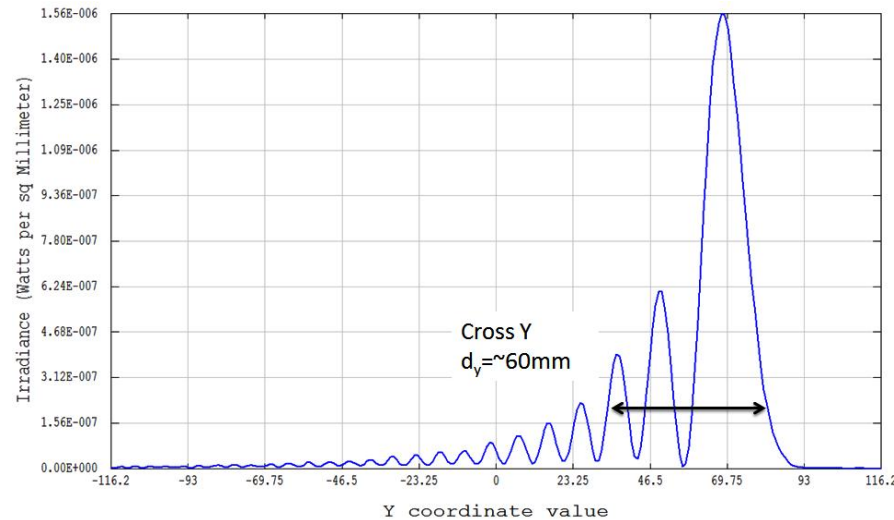

(b)

Figure 7. (a) The output beam irradiance profile at the viewing plane for the LC lens. (b) The cross section profile in the $y$ directions, the $d_{y}$ is the diameter of the beam when the irradiance value is above $1 / \mathrm{e}^{2}$.

The steering performance of the LC lens was comparable to the perfect steering lens, with the peak irradiance deflected about $68-69 \mathrm{~mm}$ from the centre of the viewing plane. The peak irradiance of the beam deflected by the LC lens is $\sim 1.6 \times 10^{-6} \mathrm{~W} / \mathrm{mm}^{2}$, slightly higher than the paraxial case of $\sim 9.8 \times 10^{-7} \mathrm{~W} / \mathrm{mm}^{2}$, but there is an issue with the ghost images caused by the input beam overfilling the LC lens. Similar diffraction issues are likely to appear when the mobile display sub-pixels overfill the LC lens, as the distance between the two layers is determined by the thickness of LC lens substrate, and this thickness is often in the order of a few hundreds micrometres. 


\section{CONCLUSION}

This work describes a pixel-level LC lenticular array structure. The LC lens pitch has approximately the same value as typical modern mobile phone sub-pixels, $\sim 20 \mu \mathrm{m}$. The phase profile of the LC lens can be changed by applying different driving signals to give different deflection angles. Such a LC lens structure can be used in many applications, for example a cylindrical lens array for phase gratings applications, and more interestingly, a time multiplexed autostereoscopic mobile display. Such a 3D display apparatus can be directly applied to an existing mobile display, and the viewers can switch between $2 \mathrm{D}$ and $3 \mathrm{D}$ views without losing the high resolution view. Future work will focus on the optimisation of the LC steering lenses, especially to reduce the crosstalk/ghost images caused by the thickness of the substrates, for a high quality auto-stereoscopic display.

\section{REFERENCES}

[1] A. Gotchev, G. B. Akar, T. Capin, D. Strohmeier, and A. Boev, "Three-Dimensional Media for Mobile Devices," Proc. IEEE, vol. 99, no. 4, pp. 708-741, Apr. 2011.

[2] J. Harrold and G. Woodgate, "Autostereoscopic display technology for mobile 3DTV applications," in Proc. SPIE 6490, 2007, vol. 6490, no. 0, pp. 1-12.

[3] N. Dodgson, “Optical devices: 3D without the glasses," Nature, vol. 495, no. 7441, pp. 316-317, 2013.

[4] D. Fattal, Z. Peng, T. Tran, S. Vo, M. Fiorentino, J. Brug, and R. G. Beausoleil, "A multi-directional backlight for a wide-angle, glasses-free three-dimensional display," Nature, vol. 495, no. 7441, pp. 348-351, Mar. 2013.

[5] Q. Mu, Z. Cao, D. Li, L. Hu, and L. Xuan, "Liquid crystal based adaptive optics system to compensate both low and high order aberrations in a model eye," Opt. Express, vol. 15, no. 4, pp. 581-587, 2007.

[6] B. Bagwell and D. Wick, "Liquid crystal based active optics," in Proc. of SPIE, 2006.

[7] Y.-P. Huang, C.-W. Chen, T.-C. Shen, and J.-F. Huang, "Autostereoscopic 3D display with scanning MultiElectrode driven liquid crystal (MeD-LC) Lens," 3D Res., vol. 1, no. 1, pp. 39-42, Aug. 2010. 